\title{
Hysteretic phenomena and switching effects under phase transitions in external field
}

\author{
L. I. Stefanovich \\ A. Galkin Physics and Engineering Institute, National Academy of Sciences of Ukraine, \\ 72 R. Luxemburg St., 340114, Donetsk, Ukraine \\ E-mail: listef@host.dipt.donetsk.ua \\ Received July 13, 1998
}

\begin{abstract}
The field dependence of the order parameter is investigated theoretically, using a statistical approach within the framework of the Ginzburg-Landau model with respect to the ferroelectric phase transition. It is found that, along with the existence of ordinary non-switching hysteresis in not too high fields $\varepsilon$ $\left(\varepsilon<<\varepsilon_{c}\right.$, where $\varepsilon_{c}$ is the coercive field), hysteresis of a switching type can be observed at the fields in excess of the coercive one.
\end{abstract}

PACS: 77.80.Dj, 77.80.Fm

The influence of a quasi-stationary external field on the formation and growth dynamics of $180^{\circ}$-domains was investigated in [1] within the framework of the Ginzburg-Landau model, using a statistical approach.

Our goal in this paper is to follow the evolution of the domain structure which has already been formed in ferroelectrics depending on the magnitude and the direction of the external field.

To characterize quantitatively the change of a system state passing through the critical temperature point $T_{c}$ of a phase transition, one or more values (called order parameters) are introduced. In the case of the ferroelectric phase transition the projection of the polarization vector on a certain crystallographic direction is used as a long-range order parameter $\eta(\mathbf{r}, t)$.

To describe the relaxation processes taking place in the system undergoing the phase transition, there is no need to know the spatial distribution of order parameter $\eta(\mathbf{r}, t)$ in detail over the total macroscopic specimen. Therefore, in the subsequent discussion, as before [1], we shall deal with the search of the main physically significant characteristics of this function, such, for example, as the average (over the crystalline grain) value of the order parameter $\langle\eta(\mathbf{r}, t)\rangle \equiv \bar{\eta}(t)$ and two-point correlation function

$$
\left\langle\xi(\mathbf{r}, t) \xi\left(\mathbf{r}^{\prime}, t\right)\right\rangle \equiv K(\mathbf{s}, t), \quad \mathbf{s}=\mathbf{r}-\mathbf{r}^{\prime},
$$

where we have introduced the centered order parameter $\xi(\mathbf{r}, t) \equiv \eta(\mathbf{r}, t)-\bar{\eta}(t)$. The equations describing the temporal evolution of $\bar{\eta}(t)$ and $K(\mathbf{s}, t)$ on all stages of ordering were introduced in [1] on the base of Ginzburg-Landau functional for nonequilibrium order parameter.

Our interest in this work is with the behavior of the order-disorder system for times which are greater in comparison with the time of forming $\left(\tau_{d}\right)$, but much less than the time of coalescence $\left(\tau_{c}\right)$ of the domain structure $\left(\tau_{d}<<t<<\tau_{c}\right)$. Therefore, in the subsequent analysis for field dependence of the order parameter $\bar{\eta}=\bar{\eta}(\varepsilon)$ we shall use the asymptotic system of equation obtained in Ref. 1

$$
\left\{\begin{array}{l}
\frac{d \bar{\eta}}{d t}=\frac{1}{2}\left[\left(\alpha-3 D(t)-\bar{\eta}^{2}\right) \bar{\eta}+\varepsilon\right], \\
\frac{d D(t)}{d t}=\left(\alpha-D(t)-3 \bar{\eta}^{2}\right) D(t),
\end{array}\right.
$$

where $D(t) \equiv K(0, t) ; \alpha=\left(T_{c}-T\right) / T_{c}$ is a dimensionless parameter which characterizes the proximity of temperature $T$, up to that the specimen cooled, to the ordering temperature $T_{c} ; \varepsilon=E / T_{c}$ is an external field in corresponding units.

The singular (stationary) points of the system (2) correspond to the thermodynamic equilibrium states of ordering system and can be found from the conditions of vanishing the first derivatives with respect to time on the left sides of the Eqs. (2) as $t \rightarrow \infty$. Hence it follows that the system of two 
algebraic equations in variables $\bar{\eta}$ and $D$ takes the form

$$
\left\{\begin{array}{l}
\varepsilon+\alpha \bar{\eta}-3 D \bar{\eta}-\bar{\eta}^{3}=0, \\
\left(\alpha-D-3 \bar{\eta}^{2}\right) D=0 .
\end{array}\right.
$$

The roots of the above-mentioned system yield the coordinates of singular points in the plane $(\bar{\eta}, D)$.

In this work we shall consider only the case $T<T_{c}$, i.e., $\alpha>0$, when there is a whole system of singular points. However, only the points located in the upper half-plane of the phase pattern (in variables $\bar{\eta}, D$ ) will have a physical sense. Recall that the positions of these singular points, in the case of weak field $\left(\varepsilon<<\alpha^{3 / 2}\right)$, were found by us in Ref. 1.

The first singular point I $[\bar{\eta}=-(\varepsilon / \alpha), D=0]$, which corresponds to a homogeneous disordered state, is an unstable node (Fig. 1).

The second point II $(\bar{\eta}=\sqrt{\alpha}+(\varepsilon / 2 \alpha), D=0)$ corresponds to a homogeneous ordered state «aligned with the external field» and is a stable node (Fig. 1).

The third point III $(\bar{\eta}=-\sqrt{\alpha}+(\varepsilon / 2 \alpha), D=0)$ corresponds to homogeneous ordering, but it is «opposite to the field». This point is also a stable node (Fig. 1).

Both the second and the third singular points correspond to single-domain type of the specimen ordering.

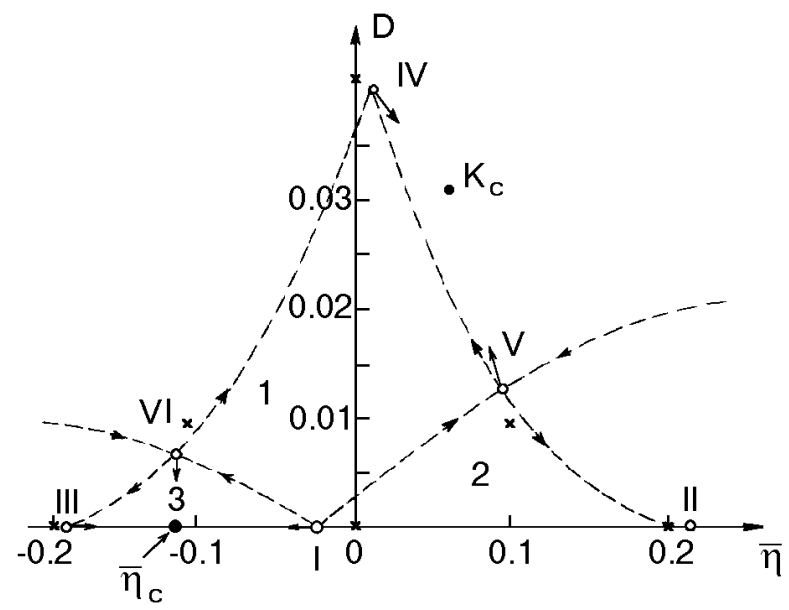

Fig. 1. Phase pattern of order-disorder system. The singular points of the system (2) at $\varepsilon=0$ are marked by the crosses $(x)$; the singular points (I-VI) for the system (2) at $\varepsilon \neq 0$ are marked by circles $(O)$ and separatrices are indicated by dotted lines. The arrows indicate the directions of motion for the singular points with increasing the external field. The bifurcation points (at $\left.\varepsilon=\varepsilon_{c}\right)$ for the system (2) $\left(\eta_{c}\right.$ and $\left.K_{c}\right)$ are shown by black circles
All the rest of the singular points (with $D \neq 0$ ) correspond to inhomogeneous ordering, i.e., to one or another polydomain structures.

The fourth point IV $\left[\bar{\eta}=\varepsilon / 2 \alpha ; D=\alpha-\left(3 \varepsilon^{2} / 4 \alpha^{2}\right)\right]$ (Fig. 1) is a stable node and corresponds to the possibility of a polydomain structure realization. The fact that $\eta \neq 0$ in this state corresponds to some non-equivalency of domains of two types. However, in weak fields this distinction is small (to the extent of the ratio $\varepsilon / \alpha$ ).

Finally, there are another two singular points of the saddle type. One of them V («right saddle») with coordinates $[\bar{\eta}=\sqrt{\alpha} / 2-\varepsilon / 4 \alpha ; D=\alpha / 4+3 \varepsilon /(4 \sqrt{\alpha})]$ (Fig. 1) corresponds to a possibility for realizing such a quasi-stationary polydomain structure, where the volume fraction of domains of the same type (e.g., with polarization vector, aligned with field) substantially exceeds the volume fraction of the other type domains (opposed to the field).

The other point ( $\ll$ left saddle») $[\bar{\eta}=-\sqrt{\alpha} / 2-$ $-\varepsilon / 4 \alpha ; D=\alpha / 4-3 \varepsilon /(4 \sqrt{\alpha})$ ] (the point VI, Fig. 1) corresponds to a possibility for realizing the quasistationary polydomain structure as well. However, in this situation the volume fraction of domains with the polarization vector oriented opposite to the field substantially exceeds a volume fraction of domains aligned with the field.

Two separatrices, leaving the origin of coordinates and passing through the «left» and «right» saddle points, divide the phase pattern into the three parts. The upper central sector (1) is the «attraction region» of inhomogeneous (polydomain) state, the lower right-hand (2) and lower left-hand sectors (3) correspond to two «attraction regions» of homogeneous single-domain states.

In the context of this work our interest will not be with the locations of singular points by itself on a phase pattern at fixed value of external field, but their evolution and bifurcation under changes of a value and direction of external field $\varepsilon$. It is suggested that the field changes slowly enough (quasistatically) in comparison with time of polydomain structure formation $\left(\tau_{\varepsilon}>>\tau_{d}\right)$. Recall that for definiteness we shall consider a phase transition in ferroelectrics connected with the appearance of a spontaneous polarization at $T<T_{c}$.

In order to follow the evolution of singular points on the phase pattern depending on the field $\varepsilon$ we direct our attention to the system (3). From the second equation of a system it follows that there are solutions of it satisfying the condition $D(t)=0$. In this case the first equation of the system (3) takes the form 


$$
\varepsilon=\bar{\eta}^{3}-\alpha \bar{\eta} .
$$

The right-hand side of (4) has extremes in two symmetric points with respect to the ordinate axis

$$
\bar{\eta}_{c}^{(1,2)}= \pm \sqrt{\alpha} / \sqrt{3} \text {. }
$$

The positive value of parameter $\left(\bar{\eta}_{c}>0\right)$ corresponds to the minimum critical field

$$
\varepsilon_{c}^{\min }=-\frac{2 \alpha^{3 / 2}}{3 \sqrt{3}}
$$

and the negative value of parameter $\left(\bar{\eta}_{c}<0\right)$ corresponds the maximum critical field

$$
\varepsilon_{c}^{\max }=\frac{2 \alpha^{3 / 2}}{3 \sqrt{3}} .
$$

Since, while writing down the thermodynamic potential (1) in [1] we assumed the at $\varepsilon>0$, then it is natural to consider only the maximum critical field $\left(\varepsilon_{c} \equiv \varepsilon_{c}^{\max }\right)$, which we shall call critical field simply. To elucidate its physical sense, we consider what will happen with the singular points of the system (2) under smooth variation of external field beginning from values $\varepsilon<<\varepsilon_{c}$.

Studying a behavior of the algebraic system (3) roots it is easy to verify that as the field $\varepsilon$ is increased, the singular points I, III and VI located in the second quadrant begin to approach each other gradually. Simultaneously, the mutual approach of the singular points IV and V occurs. And only the second singular point (II), corresponding to the homogeneous (monodomain) ordering of the system (crystallite) as a whole, will recede further and further from the rest of the singular points, moving towards the right along the abscissa axis.

The approach of the above-mentioned groups of singular points will cause the sectors 1 and 3 to decrease (Fig. 1). It testifies that the potentialities both for the polydomain and for the monodomain ordering «opposite to the field» will be decreased gradually. The sector 2 , on the contrary, grows to extend the range of conceivable initial conditions, beginning with these, the system will be ordered homogeneously, «aligned with the field». That is, the external field specifies the direction of the preferential ordering.

When the critical value of the field $\varepsilon=\varepsilon_{c}$ is achieved, the confluence of the singular points I, III and VI into a single point $\eta_{c}$ will occur. Simultaneously, i.e., at the same value of critical field, the singular points IV, $\mathrm{V}$ are merged into the singular point $K_{c}$ with the coordinates $(1 / 2 \sqrt{\alpha / 3} ; 3 \alpha / 4)$. At the same moment (i.e., in merging two groups of singular points) the sectors 1 and 3 disappear on the phase pattern (Fig. 1). In other words, at the critical value of the external field bifurcation of the ordering system trajectories takes place.

The further increasing of the external field up to the values $\varepsilon>\varepsilon_{c}$ gives all the singular points to disappear, except point II. The fields higher than critical ones should be considered to be strong. Therewith, in the range of the strong field $\left(\varepsilon>>\varepsilon_{c}\right)$, the average value of order parameter in the single-domain state does not depend on temperature to a first approximation, and it is defined only by the value of the field

$$
\bar{\eta} \sim \varepsilon^{1 / 3} .
$$

Let us consider the manner in which the character of the ferroelectric domain structure will vary under the influence of the external field. With this in mind we shall trace the variation of the order parameter average value $\bar{\eta}$ in relation to the magnitude and the direction of the applied field $\varepsilon$, identifying $\bar{\eta}$ with the average polarization of the specimen and the field $\varepsilon$ - with the uniform electric field.

Analysis will be based on treating the behavior of the solutions for the system of Eqs. (3) under slow, continuous variation of the parameter $\varepsilon$, i.e., on the study of the influence of the external field on the evolution of the ordering system singular points on the phase pattern (Fig. 1).

Initially let us take up the manner in which the ordering system which in the polydomain state corresponds to the singular point IV on the phase pattern (Fig. 1), behaves oneself with time in a weak varying external field. If the external field is changed slowly (quasi-statically) (i.e., $\tau_{\varepsilon}>>\alpha^{-1}$ ), according to the phase diagram (Fig. 1) at the small deviations of field magnitude from zero, the system is limited by the polydomain state. The external field brings into disbalance between volume fractions of the phases: oriented «opposed to the field» and «aligned with the field» in favour of the latter. In this case under small changes of the field $\left(|\varepsilon|<<\varepsilon_{c}\right)$ the order parameter $\bar{\eta}$ turns out to be proportional to the field

$$
\bar{\eta} \sim \varepsilon / \alpha .
$$

The inhomogeneities of the order parameter are therewith smoothed out by the external field

$$
D \cong \alpha-3 \varepsilon^{2} / 4 \alpha^{2} \text {. }
$$

Decreasing the dispersion of the order parameter, in accordance with (10), appears to be a second order effect in the field. 


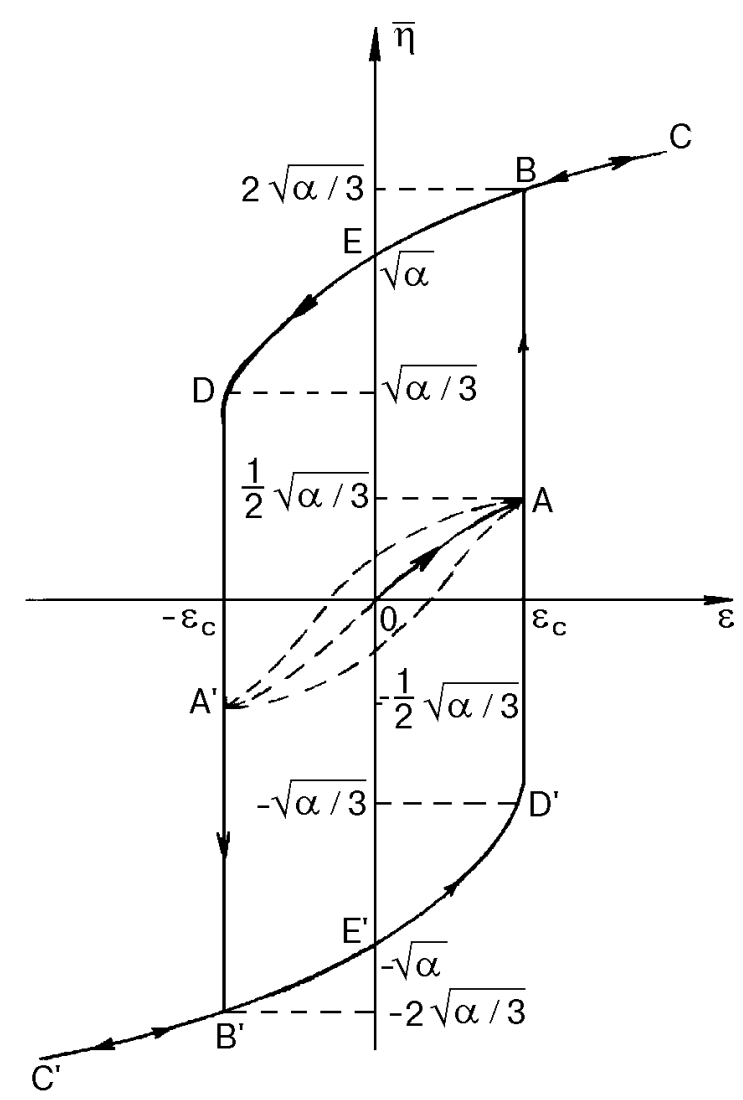

Fig. 2. Field dependences of the order parameter average value $\bar{\eta}$ : the ordinary nonswitching hysteresis is indicated by a dotted line; the hysteresis of switching type is marked by a solid line.

On further increasing the external field, as soon as the dependence $\bar{\eta}=\bar{\eta}(\varepsilon)$ ceases to obey the linear law (9), the order parameter $\bar{\eta}$ in cyclic change of the field will not follow the line OAOA'. This change of the order parameter will have the form of a hysteretic loop (dotted line in Fig. 2). The hysteresis involved (in the limits of the polydomain states) has nothing to do with the switching one and it will appear to be more significant if the amplitude of varying of the external field is larger (naturally, within the area $|\varepsilon|<\varepsilon_{c}$ ).

However such a behavior of the order parameter $\bar{\eta}$ as a function of the slowly varied external field $\varepsilon$ takes place only in the range of relatively weak field, when $|\varepsilon|<<\varepsilon_{c}$. As soon as the external field reaches its critical value $\varepsilon_{c}$, the confluence of the singular points IV and $\mathrm{V}$ on the phase pattern (Fig. 1) into a single singular point $K_{c}$, with coordinates $(1 / 2 \sqrt{\alpha / 3}, 3 \alpha / 4)$, occurs. Just at such a value of the external field $\left(\varepsilon=\varepsilon_{c}\right)$ any polydomain structure appears to be absolutely unstable with respect to further growth of field. In stronger fields $\left(\varepsilon>\varepsilon_{c}\right)$ the singular points IV, V disappear at all, that is any polydomain state becomes energetically disadvantageous and order-disorder system switches sharply (practically by a leap) to the thermodynamically stable monodomain state oriented «with the field», with the average value of the order parameter $\bar{\eta}=2 \sqrt{\alpha / 3}$ and with dispersion equal to zero $(D=0)$. This single-domain state corresponds to the singular point II on the phase pattern (Fig. 1).

Further increasing the field will cause polarization, in accordance with (8), i.e., the average value of order parameter $\bar{\eta}$ at the section of the curve from $B$ to $C$ (Fig. 2) grows insignificantly $\left(\sim \varepsilon^{1 / 3}\right)$.

The field dependence of the order parameter $\bar{\eta}=\bar{\eta}(\varepsilon)$ follows the curve $C B$ (Fig. 2) as the value of the field decreases, i.e., decreasing the average value of the order parameter up to the field value $\varepsilon=\varepsilon_{c}$ in a reversible way. However, when the field $\varepsilon$ becomes less than a critical one $\left(\varepsilon<\varepsilon_{c}\right)$, the average value of the order parameter does not return to the polydomain branch $O A$, i.e., the ordering character of a specimen remains homogeneous (monodomain). Furthermore, as the field magnitude decreases, the average value of the order parameter will continue to decrease monotonely, following the curve $B E$ (Fig. 2) and attaining the residual magnitude $\sqrt{\alpha}$ in total switching off the field $(\varepsilon=0)$. To relieve this residual value of the order parameter $\bar{\eta}$, i.e., to switch the specimen into the alternative ordering state, it is enough to change the sign of the field $\varepsilon$. In increasing the field of the opposite polarity in the range of values $-\varepsilon_{c}<\varepsilon<0$ the further decreasing of the average order parameter to the value $\sqrt{\alpha / 3}$ (the curve $E D$ on Fig. 2) will proceed. The ordering character will not be changed here until the critical value of the field $-\varepsilon_{C}$ has been attained. At the part of the curve from $E$ to $D$, when the external field $\varepsilon$ and the order parameter $\bar{\eta}$ have the opposite polarity, the states of the system are unstable thermodynamically, it would be advantageously energetically for the system, if the sign of the order parameter coincided with the field sign. In other words, the section $E D$ (as well as $E^{\prime} D^{\prime}$ ) corresponds to the metastable states of the order-disorder system.

The further increasing of the external field magnitude will produce «the frustration» of the value $\bar{\eta}$ to the magnitude $-2 \sqrt{\alpha / 3}$ (the point $B^{\prime}$, Fig. 2). This fact indicates the switching of the crystallite from the monodomain state with one direction of the spontaneous polarization into a state like this but with opposite direction of polarization in response to the electric field over the whole volume simultaneously. Thus the field $-\varepsilon_{c}$ is nothing but a coercive field. In accordance with (7), the magnitude of this coercive field depends on temperature through the parameter $\alpha=\left(T_{c}-T\right) / T_{c}$, which cha- 
racterizes a proximity of the system considered to the phase transition critical point. To evaluate the coercive field $\varepsilon_{c}$ obtained by us theoretically for really achieved values of $\alpha$, one should go to corresponding dimensional notations in (7). The transition in triglycine sulphate crystal is one of the most completely studied ferroelectric second order phase transitions. The departures of behavior answering to Landau theory are not observed in this ferroelectrics up to $\alpha \sim 10^{-4}-10^{-5}$ [2]. For these values of $\alpha$ it turns out that the magnitude of coercive field $E_{c}$ found by us will achieve quite large values $~ 50-$ $100 \mathrm{kV} / \mathrm{cm}$. However, the experimental investigations of ferroelectric hysteretic phenomena in the fields such as these or even larger ones $\left(E \sim 10^{6} \mathrm{~V} / \mathrm{cm}\right)$ are known (see, e.g., [3,4]).

The analysis of the following order parameter average value $\bar{\eta}$ behavior depending on magnitude and direction of the applied external field indicates that it is described by the curve $C^{\prime} B^{\prime} E^{\prime} D^{\prime}$ (Fig. 2), which is nothing but symmetric doubling of the line $C B E D$ with respect to the origin of the coordinates. When the field value $\varepsilon_{c}$ has been reached, the order-disorder system falls from the point $D^{\prime}$ to the point $B^{\prime}$, i.e., it switches from one monodomain state into another one. Thus, the large loop $B D B^{\prime} D^{\prime}$, known as the switching hysteresis, is closed.

However it should be borne in mind that the hysteresis pattern described theoretically above is restricted to the idealized order-disorder system. It is unlikely that «the repolarization» of the system from one monodomain state to another monodomain one (under the changes of an external field sign) will proceed over the whole volume of the specimen (crystallite) simultaneously. Actually in a volume of monodomain crystal the energetically «advantageous» regions with the opposite signs of the order parameter occur in a fluctuational way and grow rapidly [5]. In other words, along the way from one monodomain state to another monodomain one the system passes through a number of the intermediate polydomain states. Besides, different defects and imperfections of the crystalline structure give rise to the obstacles to the free motion of the domain boundaries and for their passing out of the volume. All this will make the hysteresis loop to narrow and «to smooth out», so that, for example, the coercive field may turn out to be less than the value $E_{c}$ obtained theoretically.

The author thanks to Prof. E. P. Feldman for fruitful discussions. This work was partially supported by the State Foundation of Fundamental Investigation of Ukraine, Grant No. 2. 4/220-97.

1. L. I. Stefanovich, Fizika Nizkikh Temperatur 24, 856 (1998).

2. M. E. Lines and A. M. Glass, Principles and Application of Ferroelectrics and Related Materials, Clarendon Press, Oxford (1977).

3. O. E. Fesenko, R. V. Kolesova, and Y. G. Syndeyev, Fer roelectrics 20, 177 (1978).

4. S. Sawada, T. Yamaguchi, and H. Suzuki, Ferroelectrics $\mathbf{6 3}$, 3 (1985)

5. B. A. Strukov and A. P. Levanyuk, Physical Principles of Ferroelectric Phenomena in Crystals [in Russian], Nauka, Moscow (1995). 